\title{
Spin filtering via resonant reflection of relativistic surface states
}

\author{
I. A. Nechaev ${ }^{1,2,3}$ and E. E. Krasovskii ${ }^{4,5,6}$ \\ ${ }^{1}$ Centro de Física de Materiales CFM-MPC, Centro Mixto CSIC-UPV/EHU, 20018 San Sebastián/Donostia, Spain \\ ${ }^{2}$ Tomsk State University, 634050 Tomsk, Russia \\ ${ }^{3}$ Saint Petersburg State University, 198504 Saint Petersburg, Russia \\ ${ }^{4}$ Departamento de Física de Materiales, Facultad de Ciencias Quíimicas, Universidad del Pais Vasco/Euskal Herriko Unibertsitatea, \\ Apartado 1072, San Sebastián/Donostia, 20080 Basque Country, Spain \\ ${ }^{5}$ Donostia International Physics Center (DIPC), Paseo Manuel de Lardizabal 4, San Sebastián/Donostia, 20018 Basque Country, Spain \\ ${ }^{6}$ IKERBASQUE, Basque Foundation for Science, 48013 Bilbao, Spain
}

(Received 13 September 2017; published 16 January 2018)

\begin{abstract}
A microscopic approach is developed to the scattering of surface states from a nonmagnetic linear defect at a surface with strong spin-orbit interaction. Spin-selective reflection resonances in scattering of Rashba-split surface states by an atomic stripe are theoretically discovered in a proof-of-principle calculation for a model crystal potential. Spin-filtering properties of such linear defects are analyzed within an envelope-function formalism for a perturbed surface based on the Rashba Hamiltonian. The continuous Rashba model is found to be in full accord with the microscopic theory, which reveals the essential physics behind the scattering resonance. The spin-dependent reflection suggests a novel mechanism to manipulate spins on the nanoscale.
\end{abstract}

DOI: 10.1103/PhysRevB.97.041407

Scattering of spin-orbit coupled electrons by extended defects arises in many spintronics-related phenomena, such as spin transport, accumulation, and filtering, which underlie the manipulation of spin currents in spin-based devices [1,2]. Furthermore, a detailed understanding of reflection and transmission of relativistic electrons is important for the unambiguous interpretation of scanning tunneling spectroscopy of spin-orbit split surface states $[3,4]$. A similar problem arises in ballistic transport through interfaces where powerful ab initio methods exist for scattering of bulk electrons from surfaces, such as multiple scattering [5], an embedded Green's function [6], or a Bloch-waves formalism [7]. These methods are, however, not directly applicable to scattering of surface states because of a complicated structure of the incident and reflected waves in the asymptotic (unperturbed) region. Therefore, scattering of surface states has been considered either within a tight-binding scheme [8] or within a $\mathbf{k} \cdot \mathbf{p}$ theory combined with continuity conditions for the envelope function [9-12] (see also the application to spin-dependent transport in nanowires $[13,14]$ ). However, in the $\mathbf{k} \cdot \mathbf{p}$ method the smoothness of the envelope spinor function generally conflicts with current conservation [15], which leads to a qualitatively incorrect separation of the probability current into the spin-orbit and classical-momentum contributions [16]. The tight-binding formalism, on the other hand, is not well suited for free-electron-like motion along the surface. This calls for a more universal approach to the scattering of two-dimensional (2D) states, which could be formulated in an $a b$ initio framework.

We present a method to microscopically calculate the scattering of spin-orbit split 2D states from a linear [onedimensional- (1D-) periodic] defect. Microscopic means that the system is defined by the crystal potential $V(\mathbf{r})$ and the wave functions satisfy the Schrödinger equation in real space. Therefore, the method can be straightforwardly transferred to $a b$ initio calculations. Here, we report a proof-of-principle calculation of the transmission of Rashba-split states through atomically thin defects. We study spin-filtering properties of the defects and discover spin-dependent reflection resonances for certain scatterers.

Previous studies of the effect of spin-orbit coupling on the scattering of 2D states included multibeam spin-polarized reflection from a lateral barrier [17-19], spin accumulation at the edges of semi-infinite systems [9,10,20,21], spin-selective refraction at an interface of two 2D media [22], spin-dependent transmission of electrons incident from a nonrelativistic medium through a barrier with spin-orbit coupling [11,23], and a semiclassical reflection from a smooth barrier [24]. The above studies relied on an envelope-function description of the surface states using effective Hamiltonians. By contrast, here, the perturbed surface is treated fully microscopically: The scattering problem is reduced to a supercell band-structure problem, which naturally involves both the propagating and all the required evanescent $2 \mathrm{D}$ waves and yields a detailed description of scattering, beyond the envelope-function picture. Still, the resonant properties of the scatterer can be related to the parameters of a $\mathbf{k} \cdot \mathbf{p}$ Rashba model for the perturbed surface. This demonstrates the generality of the phenomenon and suggests a way to its experimental realization.

Typical constant energy contours (CECs) of Rashba-split states comprise two circles centered at $\bar{\Gamma}$ with spin oriented along $\mathbf{k} \times \mathbf{n}$ for the inner circle (of radius $R^{+}$) and along $-\mathbf{k} \times \mathbf{n}$ for the outer circle $\left(R^{-}\right)$, where $\mathbf{n}$ is the surface normal and $\mathbf{k}$ is the $2 \mathrm{D}$ Bloch vector. We denote the unperturbed states by $\left|\mathbf{k}_{\xi}^{\chi}\right\rangle$, where $\chi= \pm$ indicates chirality and $\xi=r / t$ is the propagation direction along $x$, see Fig. 1(a). Consider a defect created by substituting a row of atoms (along the $y$ axis) by a different atom, Fig. 1(b). For a surface state $\left|\mathbf{k}_{i}^{ \pm}\right\rangle$incident from the left half-plane the scattering solution $|\Psi\rangle$ far from the defect 

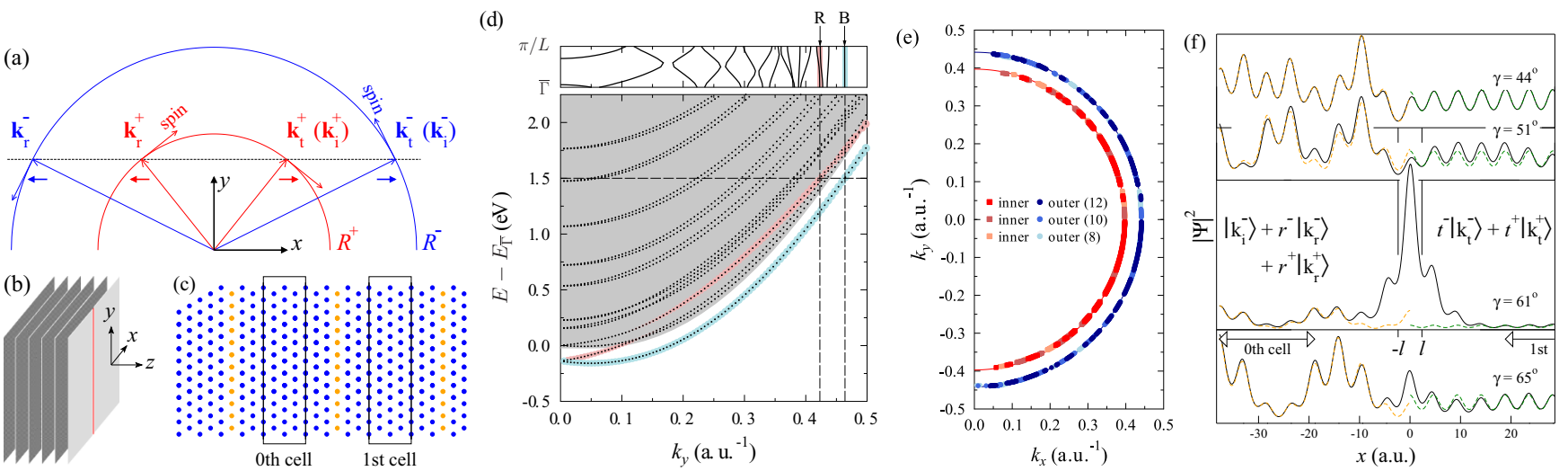

FIG. 1. (a) CECs of Rashba-split surface states. $\mathbf{k}_{\mathrm{r}}^{ \pm}, \mathbf{k}_{\mathrm{t}}^{ \pm}$, and $\mathbf{k}_{\mathrm{i}}^{ \pm}$are the Bloch vectors of reflected, transmitted, and incident waves. (b) A finite-thickness slab with a linear defect in the topmost layer. (c) Supercell geometry: topmost layer with a repeated row of impurity atoms. The two boxes indicate two asymptotic regions. (d) Upper panel: CEC at $E-E_{\bar{\Gamma}}=1.5 \mathrm{eV}$ for a 12 -fold supercell. Lower panel: The dots are the dispersion $E\left(K_{y}\right)$ of the solutions $\Phi_{\mathbf{K}^{n}}$ for $K_{x}=0$. The shaded area shows the $k_{y}$-projected states of the ideal surface. The spin-orbit split states due to the defect are highlighted in blue (true bound state) and red (resonance when inside the gray area). In the 1.5-eV CEC, the arrows indicate the bound state $(B)$ and the resonance $(R)$. (e) Bloch vectors $k_{x}$ extracted from the eigenvalues exp $(i \boldsymbol{\tau} \mathbf{k})$ of the host lattice translation operator for the three supercells for the well $(\epsilon=1.04$, upper quadrant) and the barrier $(\epsilon=0.96$, lower quadrant). (f) Density profiles of four scattering states at $E-E_{\bar{\Gamma}}=0.7 \mathrm{eV}$ for $\gamma=44^{\circ}, 51^{\circ}, 61^{\circ}$, and $65^{\circ}$ for the well $\epsilon=1.07$ in a 12 -fold supercell. The dashed lines are their asymptotic representations in the zeroth and first cells continued up to the defect.

contains two transmitted $\left|\mathbf{k}_{\mathrm{t}}^{ \pm}\right\rangle$and two reflected $\left|\mathbf{k}_{\mathrm{r}}^{ \pm}\right\rangle$waves, Fig. 1(a). The crystal momentum along $y$ is conserved, so $k_{\mathrm{t} y}^{+}=$ $k_{\mathrm{t} y}^{-}=k_{\mathrm{r} y}^{+}=k_{\mathrm{r} y}^{-}=k_{\mathrm{i} y}^{\chi}$. For $R^{-}>k_{\mathrm{i} y}^{-}>R^{+}$there is only one transmitted and one reflected wave. In the unperturbed region, $|\Psi\rangle$ contains also evanescent waves, and depending on how fast they decay away from the defect the scattering state $|\Psi\rangle$ can be obtained from band-structure solutions $\Phi_{\mathbf{K}^{n}}$ for a smaller or larger supercell, Fig. 1(c). The method works as follows: At a given $k_{i y}^{\chi}$, the surface states perturbed by the periodic defect give rise to four (or two) supercell eigenfunctions $\Phi_{\mathbf{K}^{n}}$ with the supercell crystal momentum $K_{y}^{n}=k_{\mathrm{i} y}^{\chi}, n=1-4(n=$ 1,2 if $k_{\mathrm{i} y}^{-}>R^{+}$), see Fig. 1(d). Far from the scatterers [in the asymptotic region denoted the zeroth cell in Fig. 1(c)] the functions $\Phi_{\mathbf{K}^{n}}(\mathbf{r})$ can obviously be decomposed into a sum of the four unperturbed surface states $\left|\mathbf{k}_{\xi}^{\chi}\right\rangle$. The latter are obtained as eigenfunctions of the translation operator of the ideal surface $\hat{T} \Theta(\mathbf{r})=\Theta(\mathbf{r}+\boldsymbol{\tau})=\exp (i \boldsymbol{\tau} \mathbf{k}) \Theta(\mathbf{r})$ in terms of the supercell solutions: $\Theta=\sum_{n} c^{n} \Phi_{\mathbf{K}^{n}}$. The functions $\Theta$ are defined everywhere in the crystal, and in the asymptotic region they coincide with the unperturbed surface states: $\Theta_{\xi}^{\chi}(\mathbf{r})=$ $\left\langle\mathbf{r} \mid \mathbf{k}_{\xi}^{\chi}\right\rangle$. The full scattering solution is then a linear combination $\Psi=\sum_{\chi \xi} a_{\xi}^{\chi} \Theta_{\xi}^{\chi}$ defined by the condition that $\Psi$ contains only one right-traveling wave in the zeroth supercell and no left-traveling waves in the first supercell (the next asymptotic region), Fig. 1(c). Note that $\Psi$ is valid everywhere, including the vicinity of the defect.

Let us consider a seven-layer slab with the geometry of a $\mathrm{Au}(111)$ surface with an overlayer. The atoms are represented by a three-dimensional (3D) regular muffin-tin potential, which is expanded in a truncated 3D Fourier series and included into the microscopic Hamiltonian $\hat{p}^{2}+V(\mathbf{r})+\beta \boldsymbol{\sigma} \cdot[\nabla V(\mathbf{r}) \times \hat{\mathbf{p}}]$ with $\beta$ scaled such that the Rashba splitting of the surface states be close to that in $\mathrm{Au}(111)$. The supercell band structure is calculated on a rectangular $\mathbf{k}$ mesh with $\Delta K_{x}=\Delta K_{y}=$ 0.0056 a.u. ${ }^{-1}$, and the functions $\Phi_{\mathbf{K}^{n}}$ for a given energy and $K_{y}$ are obtained by triangular interpolation. A typical constant energy contour is shown in the upper panel of Fig. 1(d). The artificial periodicity of the defect gives rise to spectral gaps, so for certain $K_{y}$ there are no solutions $\Phi$. However, for a given $K_{y}$ one can always choose a supercell for which the solution exists.

We will consider two types of defects: barrier and well. For a barrier, the potential at the impurity site $U_{\mathrm{D}}$ is shallower than the potential $U_{\mathrm{S}}$ at the host atom, and for a well it is deeper. Computationally, the muffin-tin potentials $U_{\mathrm{D}}$ are linearly scaled: $U_{\mathrm{D}}(r)=\epsilon U_{\mathrm{S}}(r)$. The Bloch vectors of the unperturbed surface states extracted from the eigenvalues $\exp (i \boldsymbol{\tau} \mathbf{k})$ are shown in Fig. 1(e). The good agreement between the three supercells both for a well and for a barrier demonstrates that the evanescent waves are negligible in the asymptotic region already for the eightfold supercell. Figure 1(f) shows the density profiles of the outer-circle surface states at $E-E_{\bar{\Gamma}}=$ $0.7 \mathrm{eV}$ scattered by a well $\epsilon=1.07$ for four angles of incidence $\gamma$. Although the unperturbed surface states are derived from the zeroth cell, the asymptotic representation is seen to be valid over a much wider region (see, especially, $\gamma=44^{\circ}$ ). The transmission probability $T^{ \pm}$as a function of $\gamma$ is shown in Fig. 2 for a barrier $\epsilon=0.96$ and for two wells $\epsilon=1.04$ and 1.07. Here $T^{+}$and $T^{-}$stand for the incident wave in the inner and in the outer circles, respectively. The colored symbols are the microscopic calculations with shades of red used for $T^{+}$and shades of blue used for $T^{-}$. The voids in the curves correspond to the gaps in the supercell band structure, and in approaching the gap the transmission sometimes shows a spurious growth [see the vertical arrows in Figs. 2(a) and 2(b)]. This happens when two of the Bloch vectors $K_{y}^{n}$ are close to the edge of the Brillouin zone and the numerical method finds the two solutions $\Phi_{\mathbf{K}^{n}}$ linearly dependent. Such artifacts are recognized by an accuracy criterion, and they are easily sorted out because they occur at different angles for different supercells.

Most important is the strikingly different behavior of the transmission probability $T^{-}$for the two types of defects: for a 


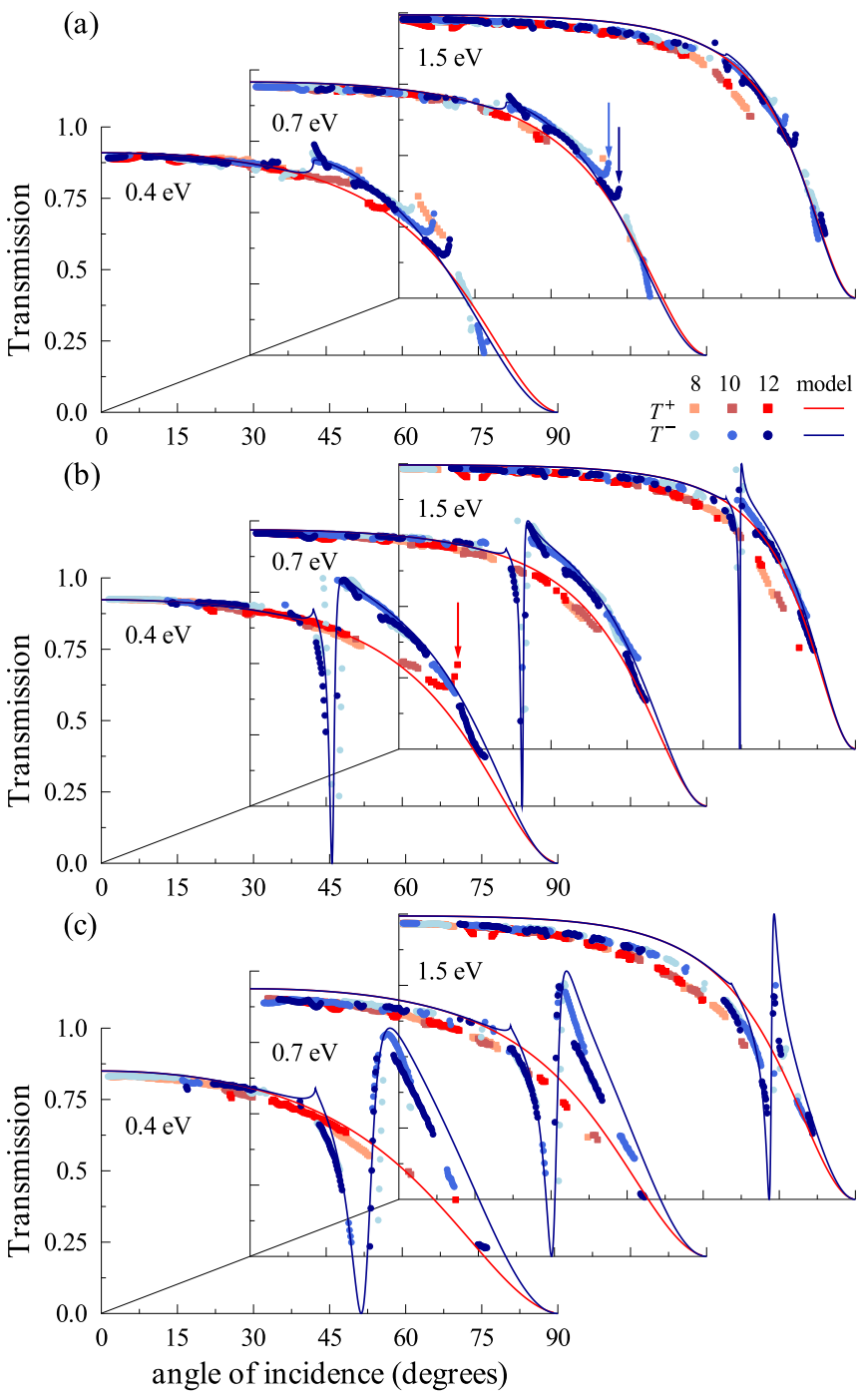

FIG. 2. Transmission probability as a function of the angle of incidence for the (a) $\epsilon=0.96$, (b) 1.04 , and (c) 1.07 for $E-E_{\bar{\Gamma}}=$ $1.5,0.7$, and $0.4 \mathrm{eV}$. The shades of red (blue) show $T^{+}\left(T^{-}\right)$for eightfold, tenfold, and 12-fold supercells. The solid lines are the continuous model fit of $T^{+}$(red) and $T^{-}$(blue) obtained with $U=0.27,-0.33$, and $-0.55 \mathrm{eV}$ for $\epsilon=0.96,1.04$, and 1.07 , respectively. The parameters $\alpha$ and $m^{*}$ for the presented energies are listed in Table I.

barrier, $T^{-}$steadily decreases, Fig. 2(a), whereas for a well, it shows a sharp minimum followed by a maximum, see Figs. 2(b) and 2(c). By contrast, $T^{+}$steadily decreases in both cases. To understand this behavior, let us consider the contribution of evanescent waves to the scattering states $\Psi$. Their weight can be inferred from the deviation of the density profile $|\Psi|^{2}$ in Fig. 1(f) from the left and right asymptotics (the dashed lines) continued up to the scatterer. For small angles the weight of the evanescent waves is negligible, and it starts growing when $k_{\mathrm{i} y}^{-}$ exceeds $R^{+}$because the evanescent waves replace the missing propagating solutions of the inner circle. This point manifests itself by a cusp maximum in $T^{-}$, e.g., at $0.4 \mathrm{eV}$ around $\gamma=$ $45^{\circ}$ in Fig. 2(a). In approaching the minimum [see $\gamma=61^{\circ}$ in Fig. 1(f)] the density around the defect steeply grows and
TABLE I. Rashba Hamiltonian parameters $m^{*}$ and $\alpha$ used to model the transmission through the defect, Fig. 2. They are derived by fitting the dispersion $E(k)$ of the unperturbed surface state of the microscopic calculation. $m^{*}$ and $\alpha$ depend on energy because $E(k)$ is not exactly parabolic. Atomic Hartree units are used: $\hbar=m_{0}=$ $e=1$.

\begin{tabular}{lccc}
\hline \hline$E-E_{\bar{\Gamma}}(\mathrm{eV})$ & $m^{*}($ a.u. $)$ & $\alpha$ (a.u.) & $R^{-}-R^{+}\left(\right.$a.u. $\left.^{-1}\right)$ \\
\hline 0.0 & 1.59 & 0.033 & 0.105 \\
0.4 & 1.85 & 0.026 & 0.096 \\
0.7 & 2.00 & 0.020 & 0.080 \\
1.5 & 2.00 & 0.012 & 0.048 \\
\hline \hline
\end{tabular}

then rapidly decreases with increasing $\gamma$. This happens because the defect causes a sharp perturbation of the potential $V(\mathbf{r})$ (comparable to the lattice period), which is known to give rise to a bound state localized at the defect and energetically split off from the band continuum [25]. For the Rashba states that are bounded only from below a barrier does not produce any bound states. By contrast, a well-like perturbation produces two structures [Fig. 1(d)]: bound state $B$ and its spin-orbit counterpart resonance $R$ [highlighted red in Fig. 1(d)]. The hybridization of the incident wave of the outer circle with the resonance- the inner branch of the Rashba-split 1D impurity state-gives rise to the asymmetric $T^{-}(\gamma)$ feature.

In order to relate the reflection resonance to phenomenologically relevant spin-orbit characteristics of the material let us consider a Rashba system with the Hamiltonian $\hat{H}_{\mathrm{R}}=$ $k^{2} / 2 m^{*}+\alpha\left(k_{y} \sigma_{x}-k_{x} \sigma_{y}\right)$. The relation $k^{2}=k_{x}^{2}+k_{y}^{2}$ determines whether a given branch is propagating or evanescent for a given $k_{y}$ and $E$ [11]. The defect is represented by a potential barrier (well) $V(x)=U$ for $-l<x<l$ with $V(x)=0$ elsewhere. The width of the defect equals the width of the unit cell: $2 l=\tau_{x}$. The scattering solution is found by the condition of the continuity of the spinor wave function and flux across the defect [15]. Thus, the envelope-function formalism solves the problem without resorting to an artificial supercell. A four-wave representation in the perturbed region $\sum_{\chi \xi} d_{\xi}^{\chi}\left|\tilde{\mathbf{k}}_{\xi}^{\chi}\right\rangle$ is matched to the wave function in the left and right half-planes at the boundaries $x= \pm l$ indicated in Fig 1(f). Here $\tilde{\mathbf{k}}$ 's are the wave vectors of the eigenfunctions of the Hamiltonian $\hat{H}_{\mathrm{R}}+U$ (with the same $\alpha$ and $m^{*}$ as for the unperturbed surface). The scattering problem then reduces to an $8 \times 8$ matrix equation $\hat{M} \mathbf{a}=\mathbf{f}$ for the vector $\mathbf{a}=\left(r^{ \pm}, d_{\mathrm{r}}^{ \pm}, d_{\mathrm{t}}^{ \pm}, t^{ \pm}\right)^{\mathrm{T}}$. Here $\hat{M}$ is the matching matrix, and $r^{ \pm}$and $t^{ \pm}$are the coefficients of the two reflected and two transmitted waves in the two unperturbed half-planes, see the legends in Fig. 1(f). The right-hand side f represents the incident wave $\left|\mathbf{k}_{\mathrm{i}}^{\chi}\right\rangle$, and it has four nonzero components: the value and the flux (for both spins) at $x=-l$.

The transmitted current $T^{\chi}=\left|t_{\chi}^{+}\right|^{2}+\left|t_{\chi}^{-}\right|^{2}$ is shown in Fig. 2 by the solid lines. With $U$ adjusted to fit the microscopic calculations the Rashba model perfectly reproduces the shape of the curves and the dependence of the position and the width of the resonance on the energy and on the scatterer. Surprisingly, the envelope-function method originally designed for slowly varying potentials shows excellent performance for the atomic stripe. To establish the analogy with the microscopic picture, let us consider the eigenspectrum of the perturbed 

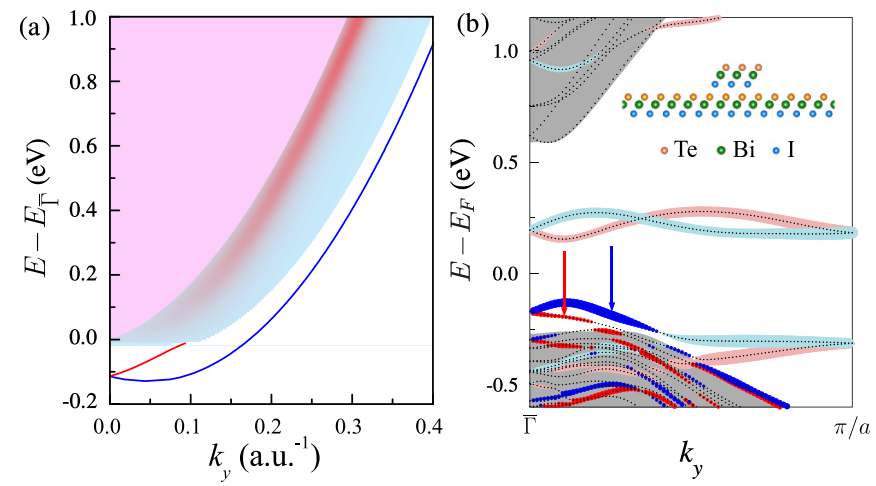

FIG. 3. (a) Electronic structure of the Rashba system with linear defect. The solid lines show the bound states split off from the Rashba continuum. The width of the blurred red line shows the $k_{y}$ width of the resonance. The Rashba continuum of the ideal system $E^{ \pm}(\mathbf{k})[\mathrm{cf}$. the gray area in Fig. 1(d)] is shown by the sign of the $k_{y}$-projected $x$-spin spectral density $S_{x}^{\text {tot }}$. Light red indicates $S_{x}^{\text {tot }}>0$, and light blue indicates $S_{x}^{\text {tot }}<0$. (b) Band structure and side-view geometry of a BiTeI trilayer with a nanostripe. The shaded area covers the $k_{y}$-projected states of the clean trilayer. The localization of the trilayer states on the atoms beneath the stripe are shown by red $\left(\sigma_{x}^{\uparrow}\right)$ and blue $\left(\sigma_{x}^{\downarrow}\right)$ fat bands. Light red (light blue) fat bands correspond to $\sigma_{x}^{\uparrow}\left(\sigma_{x}^{\downarrow}\right)$ dangling bond states localized on the stripe.

system. It is obtained by dropping the incident wave and finding zeros of the real and imaginary parts of the determinant of the matrix $\hat{M}$. In Fig. 3(a), the ideal surface is presented by the energy-momentum distribution of the sign ( $\uparrow$ or $\downarrow$ ) of the $k_{y}$-projected $\sigma_{x}$-spin spectral density $S_{x}^{\text {tot }}=S_{x}^{+}+S_{x}^{-}$, where $S_{x}^{ \pm}\left(E, k_{y}\right)=\int d k_{x}\left\langle\mathbf{k}^{ \pm}\left|\sigma_{x}\right| \mathbf{k}^{ \pm}\right\rangle \delta\left[E-E^{ \pm}(\mathbf{k})\right] / 8 \pi^{2}$. The incident wave comes from the $\sigma_{x}^{\downarrow}$ continuum (blue area), which overlaps with the spectral resonance having $\sigma_{x}^{\uparrow}$ spin. Just at the resonance, $T^{-}(\gamma)$ sharply drops to zero and then steeply rises to unity, exactly as in the microscopic model, see Figs. 2(b) and 2(c). Thus, the scattering by the 1D defect is transparently expressed through the relation between the CECs of the host and the defect region. Here, an important ingredient is the spin nonconservation, so the effect does not occur, e.g., for Zeeman splitting.

Finally, as a possible platform for the experimental realization of the discovered resonant reflection, we suggest the layered semiconductors of the $\operatorname{BiTe} X(X=\mathrm{I}, \mathrm{Br}, \mathrm{Cl})$ family where giant Rashba-split surface states reside in an absolute gap [26-30]. Already a trilayer Te-Bi- $X$-the easily exfoliated structure element of these semiconductors-provides the desired 2D spin-orbit split valence and conduction states [31,32]. For holes and electrons of a stand-alone trilayer, a perturbation can be introduced by putting on it a nanostripe as shown in the inset of Fig. 3(b) [33]. This linear defect gives rise to 1D spin-orbit split bound states that split off from the valence band as follows from our $a b$ initio calculation [34], see the vertical arrows in Fig. 3(b). These states form the 1D Rashba channel that guides the holes. As seen in Fig. 3(b), the inner branch (the red arrow) becomes the above-mentioned spectral resonance when it enters the projected continuum.

To summarize, we have developed a microscopic approach for the scattering of relativistic surface states by a linear defect and found strong spin selectivity of electron transmission for well-like perturbations. Thereby, the transmitted spin current can be enhanced, which suggests a potential technique for nonmagnetic spin filtering and spin injection.

This work was supported by the Spanish Ministry of Economy and Competitiveness MINECO (Project No. FIS201676617-P). I.A.N. also acknowledges support from Saint Petersburg State University (Grant No. 15.61.202.2015).
[1] S. A. Wolf, D. D. Awschalom, R. A. Buhrman, J. M. Daughton, S. von Molnár, M. L. Roukes, A. Y. Chtchelkanova, and D. M. Treger, Spintronics: A spin-based electronics vision for the future, Science 294, 1488 (2001).

[2] D. Bercioux and P. Lucignano, Quantum transport in Rashba spinorbit materials: A review, Rep. Prog. Phys. 78, 106001 (2015).

[3] L. El-Kareh, P. Sessi, T. Bathon, and M. Bode, Quantum Interference Mapping of Rashba-Split Bloch States in Bi/Ag(111), Phys. Rev. Lett. 110, 176803 (2013).

[4] S. Schirone, E. E. Krasovskii, G. Bihlmayer, R. Piquerel, P. Gambardella, and A. Mugarza, Spin-Flip and Element-Sensitive Electron Scattering in the BiAg 2 Surface Alloy, Phys. Rev. Lett. 114, 166801 (2015).

[5] J. Henk, A. Ernst, K. K. Saha, and P. Bruno, Computing conductances of tunnel junctions by the Korringa-Kohn-Rostoker method: Formulation and test of a Green function approach, J. Phys.: Condens. Matter 18, 2601 (2006).

[6] D. Wortmann, H. Ishida, and S. Blügel, Embedded Green-function approach to the ballistic electron transport through an interface, Phys. Rev. B 66, 075113 (2002).
[7] E. E. Krasovskii, Augmented-plane-wave approach to scattering of Bloch electrons by an interface, Phys. Rev. B 70, 245322 (2004).

[8] K. Kobayashi, Electron transmission through atomic steps of $\mathrm{Bi}_{2} \mathrm{Se}_{3}$ and $\mathrm{Bi}_{2} \mathrm{Te}_{3}$ surfaces, Phys. Rev. B 84, 205424 (2011).

[9] G. Usaj and C. A. Balseiro, Spin accumulation and equilibrium currents at the edge of 2DEGs with spin-orbit coupling, Europhys. Lett. 72, 631 (2005).

[10] A. Reynoso, G. Usaj, and C. A. Balseiro, Spin hall effect in clean two-dimensional electron gases with Rashba spin-orbit coupling, Phys. Rev. B 73, 115342 (2006).

[11] V. A. Sablikov and Y. Y. Tkach, Evanescent states in twodimensional electron systems with spin-orbit interaction and spin-dependent transmission through a barrier, Phys. Rev. B 76, 245321 (2007).

[12] H. Xie, F. Jiang, and W. E. I. Sha, Numerical methods for spin-dependent transport calculations and spin bound states analysis in Rashba waveguides, Comput. Phys. Commun. 198, 118 (2016).

[13] L. Zhang, P. Brusheim, and H. Q. Xu, Multimode electron transport through quantum waveguides with spin-orbit interaction 
modulation: Applications of the scattering matrix formalism, Phys. Rev. B 72, 045347 (2005).

[14] M. I. Alomar, L. Serra, and D. Sánchez, Interplay between resonant tunneling and spin precession oscillations in all-electric all-semiconductor spin transistors, Phys. Rev. B 94, 075402 (2016).

[15] L. W. Molenkamp, G. Schmidt, and G. E. W. Bauer, Rashba Hamiltonian and electron transport, Phys. Rev. B 64, 121202 (2001).

[16] E. E. Krasovskii, Microscopic origin of the relativistic splitting of surface states, Phys. Rev. B 90, 115434 (2014).

[17] A. O. Govorov, A. V. Kalameitsev, and J. P. Dulka, Spindependent transport of electrons in the presence of a smooth lateral potential and spin-orbit interaction, Phys. Rev. B 70, 245310 (2004).

[18] H. Chen, J. J. Heremans, J. A. Peters, A. O. Govorov, N. Goel, S. J. Chung, and M. B. Santos, Spin-polarized reflection in a two-dimensional electron system, Appl. Phys. Lett. 86, 032113 (2005).

[19] V. Teodorescu and R. Winkler, Spin angular impulse due to spin-dependent reflection off a barrier, Phys. Rev. B 80, 041311 (2009).

[20] E. B. Sonin, Edge spin accumulation: Spin Hall effect without bulk spin current, Phys. Rev. B 81, 113304 (2010).

[21] A. Khaetskii and E. Sukhorukov, Unitarity of scattering and edge spin accumulation, Phys. Rev. B 87, 075303 (2013).

[22] M. Khodas, A. Shekhter, and A. M. Finkel'stein, Spin Polarization of Electrons by Nonmagnetic Heterostructures: The Basics of Spin Optics, Phys. Rev. Lett. 92, 086602 (2004).

[23] V. M. Ramaglia, D. Bercioux, V. Cataudella, G. De Filippis, and C. A. Perroni, Spin polarization of electrons with Rashba double-refraction, J. Phys.: Condens. Matter 16, 9143 (2004).

[24] P. G. Silvestrov and E. G. Mishchenko, Polarized electric current in semiclassical transport with spin-orbit interaction, Phys. Rev. B 74, 165301 (2006).

[25] Otfried Madelung, Introduction to Solid-State Theory (SpringerVerlag, Berlin, Heidelberg, 1978).

[26] K. Ishizaka, M. S. Bahramy, H. Murakawa, M. Sakano, T. Shimojima, T. Sonobe, K. Koizumi, S. Shin, H. Miyahara, A. Kimura, K. Miyamoto, T. Okuda, H. Namatame, M. Taniguchi, R. Arita, N. Nagaosa, K. Kobayashi, Y. Murakami, R. Kumai,
Y. Kaneko, Y. Onose, and Y. Tokura, Giant Rashba-type spin splitting in bulk BiTeI, Nature Mater. 10, 521 (2011).

[27] A. Crepaldi, L. Moreschini, G. Autès, C. Tournier-Colletta, S. Moser, N. Virk, H. Berger, P. Bugnon, Y. J. Chang, K. Kern, A. Bostwick, E. Rotenberg, O. V. Yazyev, and M. Grioni, Giant Ambipolar Rashba Effect in the Semiconductor BiTeI, Phys. Rev. Lett. 109, 096803 (2012).

[28] S. V. Eremeev, I. A. Nechaev, Y. M. Koroteev, P. M. Echenique, and E. V. Chulkov, Ideal Two-Dimensional Electron Systems with a Giant Rashba-Type Spin Splitting in Real Materials: Surfaces of Bismuth Tellurohalides, Phys. Rev. Lett. 108, 246802 (2012).

[29] M. Sakano, M. S. Bahramy, A. Katayama, T. Shimojima, H. Murakawa, Y. Kaneko, W. Malaeb, S. Shin, K. Ono, H. Kumigashira, R. Arita, N. Nagaosa, H. Y. Hwang, Y. Tokura, and K. Ishizaka, Strongly Spin-Orbit Coupled Two-Dimensional Electron Gas Emerging Near the Surface of Polar Semiconductors, Phys. Rev. Lett. 110, 107204 (2013).

[30] S. V. Eremeev, I. P. Rusinov, I. A. Nechaev, and E. V. Chulkov, Rashba split surface states in BiTeBr, New J. Phys. 15, 075015 (2013).

[31] Y. L. Chen, M. Kanou, Z. K. Liu, H. J. Zhang, J. A. Sobota, D. Leuenberger, S. K. Mo, B. Zhou, S.-L. Yang, P. S. Kirchmann, D. H. Lu, R. G. Moore, Z. Hussain, Z. X. Shen, X. L. Qi, and T. Sasagawa, Discovery of a single topological Dirac fermion in the strong inversion asymmetric compound $\mathrm{BiTeCl}$, Nat. Phys. 9, 704 (2013).

[32] Y. Ma, Y. Dai, W. Wei, X. Li, and B. Huang, Emergence of electric polarity in BiTeX $(\mathrm{X}=\mathrm{Br}$ and $\mathrm{I})$ monolayers and the giant Rashba spin splitting, Phys. Chem. Chem. Phys. 16, 17603 (2014).

[33] We consider a stripe of 1-nm width made of a single BiTeI trilayer and with stable stoichiometric edges as in Ref. [35].

[34] Our density-functional-theory calculations within the generalized-gradient approximation employed the full-potential linearized augmented-plane-wave method implemented in the FLEUR code, http://www.flapw.de.

[35] S. V. Eremeev, I. A. Nechaev, and E. V. Chulkov, Two- and three-dimensional topological phases in $\operatorname{BiTe} X$ compounds, Phys. Rev. B 96, 155309 (2017). 